\title{
Forced oscillations of coronal loops driven by EIT waves ${ }^{\star}$
}

\author{
I. Ballai ${ }^{1}$, M. Douglas ${ }^{1}$, and A. Marcu ${ }^{2}$ \\ ${ }^{1}$ Solar Physics and Space Plasma Research Centre (SP2 RC), Dept. of Applied Mathematics, University of Sheffield, Hicks Building, \\ Hounsfield Road, Sheffield, S3 7RH, UK \\ e-mail: i.ballai@sheffield.ac.uk \\ 2 Babes-Bolyai University, Dept. of Theoretical and Computational Physics, 1 Kogalniceanu, 400084 Cluj-Napoca, Romania
}

Received 24 March 2008 / Accepted 9 July 2008

\begin{abstract}
Aims. We study the generation of transversal oscillations in coronal loops represented as a straight thin flux tube under the effect of an external driver modelling the global coronal EIT wave. We investigate how the generated oscillations depend on the nature of the driver, and the type of interaction between the two systems.

Methods. We consider the oscillations of a magnetic straight cylinder with fixed-ends under the influence of an external driver modelling the force due to the global EIT wave. Given the uncertainties related to the nature of EIT waves, we first approximate the driver by an oscillatory force in time and later by a shock with a finite width.

Results. Results show that for a harmonic driver the dominant period in the generated oscillation belongs to the driver. Depending on the period of driver, compared to the natural periods of the loop, a mixture of standing modes harmonics can be initiated. In the case of a non-harmonic driver (modelling a shock wave), the generated oscillations in the loop are the natural periods only. The amplitude of oscillations is determined by the position of the driver along the tube. The full diagnosis of generated oscillations is achieved using simple numerical methods.
\end{abstract}

Key words. magnetohydrodynamics (MHD) - Sun: corona - waves

\section{Introduction}

Latest high-resolution coronal observations have shown that coronal structures are very dynamic entities with flows and waves propagating along them. Waves and oscillations in coronal loops have received increased attention in the last few years due to the possibility to use the observed properties to diagnose not only the magnetic field in these structures, but also the subresolution space distribution of loops, plasma properties, etc. (e.g. Roberts et al. 1984; Aschwanden et al. 1999; Nakariakov et al. 1999; Banerjee et al. 2007; Verth et al. 2007; Arregui et al. 2008; Verth \& Erélyi 2008). One specific type of oscillations observed in coronal loops is the fast kink mode which disturbs the symmetry axis of the loop (for a full description of these waves see, e.g. Edwin \& Roberts 1983) and it is nearly incompressible.

Many of kink waves and oscillations have their origin in the interaction of coronal loops with various external sources and drivers (see, e.g. Hindman \& Jain 2008; Erdélyi \& Hargreaves 2008). One of the possible explanations of oscillations in coronal loops and/or prominence fibrils is that they have their origin in the interaction of these loops with global coronal waves, e.g. EIT waves. EIT waves (Thompson et al. 1999) are waves generated by sudden energy releases (flares, coronal mass ejections, etc.) and they are able to propagate over very large distances in the solar low corona.

Observational evidence for large-scale coronal impulses initiated during the early stage of a flare and/or CME has been provided by the EIT instrument onboard SOHO, TRACE/EUV,

^ EIT: Extreme-Ultraviolet Imaging Telescope.
STEREO/EUVI. EIT waves propagate in the quiet Sun with speeds of $250-400 \mathrm{~km} \mathrm{~s}^{-1}$ at an almost constant altitude. At a later stage in their propagation EIT waves can be considered as a freely propagating wavefront which is observed to interact with coronal loops (see, e.g. Wills-Davey \& Thompson 1999). Using TRACE/EUV $195 \AA$ ̊ observations, Ballai et al. (2005) have shown that EIT waves - seen in this wavelength - are waves with average periods of the order of $400 \mathrm{~s}$. Since at the height where the EUV lines are formed, the magnetic field can be considered vertical, EIT waves were interpreted as fast magnetohydrodynamic (MHD) waves. This interpretation was confirmed using multi-wavelength STEREO/EUVI observations by Long et al. (2008). Recently, Attrill et al. (2007a,b) proposed that the diffuse EIT coronal bright fronts are due to driven magnetic reconnections between the skirt of the expanding CME magnetic field and the favorably orientated quiet Sun magnetic field. According to this latter model, the propagation process of the front consists of a sequence of successive reconnection events.

Although a large consensus was reached on the trigger mechanism of these global coronal waves and effects EIT waves can generate, the nature of these large scale disturbances is still unknown, despite the multitudes of models. The main reason of this uncertainty is the lack of high temporal and spatial resolution as well as the limited field of view (in the case of TRACE/EUV).

The present paper investigates the temporal and spatial variations of transversal oscillations in a coronal loop under the influence of an external driver representing the coronal global EIT waves. Strictly speaking a coronal loop application would require the consideration of an external magnetic field. However, we consider this study as a starting point in a much more 


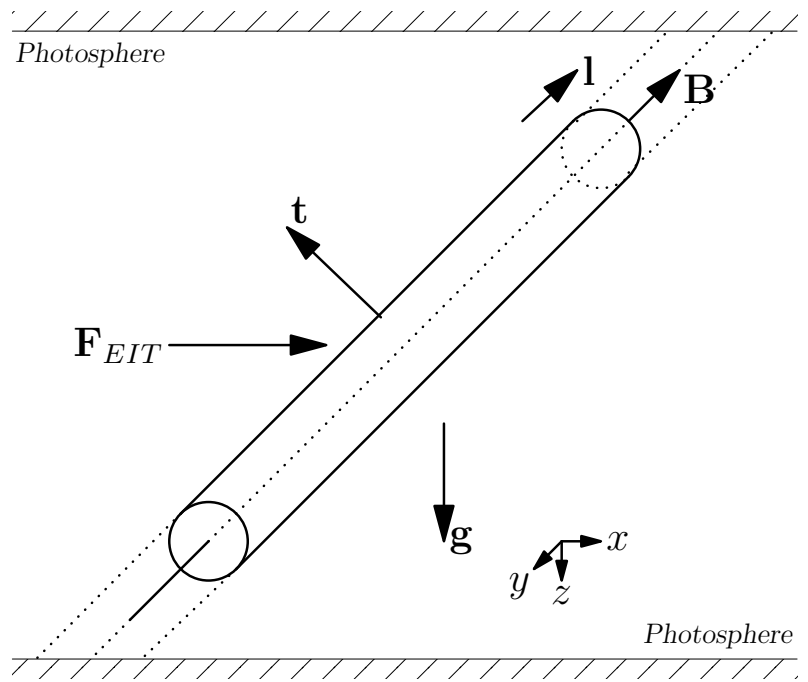

Fig. 1. A schematic representation of the working model. The straight flux tube, arbitrarily inclined with respect to the vertical direction, is under the influence of different forces which will generate transversal oscillations in the coronal loop which has fixed ends in the dense photosphere.

complex analysis. Due to the uncertainties in resolving the nature of EIT waves, we will discuss separately the cases of a harmonic driver and a driver of a finite width with a pulse-like temporal distribution. It should be noted here that the study of the present papers apply not only to EIT waves as external driver, but it could be applied to any external source.

In the next section we introduce the working model and derive the governing equation of transversal oscillations based on the principle of force equilibrium in conjunction with the continuity of mass and magnetic flux. The governing equation is solved for the a driving force when its particular form is not specified. Section 3 is devoted to the study of the periodical motion of the coronal loop under the influence of a few particular drivers. Finally, in Sect. 4 we summarize our results and discuss some possible key extensions which were neglected here but they could be added to this model in subsequent studies.

\section{Wave equations and solutions}

Let us suppose that a flux tube is situated in a magnetic free environment and it is under the effect of gravity. This model could approximate (in the first order) a magnetic loop in the solar corona. We consider the magnetic rope in the thin-fluxtube approximation inclined at an arbitrary angle with respect to the vertical. Let us suppose the directions $\boldsymbol{l}$ and $\boldsymbol{t}$ to be oriented along the loop and in a transversal direction. We assume that the EIT wave is acting with a force $\boldsymbol{F}$ (per unit volume) on the tube and the direction of this force is directed along the $x$-axis. For the sake of simplicity all dissipative effects are neglected. A schematic representation of the model is shown in Fig. 1. Part of the discussion of this paper is using the model and derivation developed by Spruit (1981) where the transversal waves were studied in a magnetic flux tube in the convective zone/photosphere.

Any vector $\boldsymbol{a}$ can be decomposed with respect to the parallel and perpendicular direction of the tube such as

$a_{\|}=(\hat{l} \cdot a) \hat{l}, \quad a_{\perp}=(\hat{l} \times a) \times \hat{l}$.

The forces acting on the tube are the pressure force, the Lorentz force, the gravitational force and the force from the EIT wave.
These forces are going to be decomposed along the two characteristic directions. We suppose that the homogeneous magnetic field is untwisted and it has a single component, along the tube, i.e. $\boldsymbol{B}=B \hat{\boldsymbol{l}}$.

The parallel component of the motion is driven by the parallel components of acting forces. Since the Lorentz force has no field aligned component, it will appear only in the perpendicular direction. The parallel force equilibrium requires that

$\rho_{\mathrm{i}}\left(\frac{\mathrm{d} \boldsymbol{v}}{\mathrm{d} t}\right)_{\|}=-\partial_{\mathrm{l}} p+\rho_{\mathrm{i}} \boldsymbol{g} \cdot \hat{\boldsymbol{l}}+\boldsymbol{F} \cdot \hat{\boldsymbol{l}}$

where the operator $\partial_{1}$ is defined as $\partial_{1}=\hat{\boldsymbol{l}} \cdot \nabla$ and $\rho_{\mathrm{i}}$ is the density inside the loop. Along the perpendicular direction the forces acting on the tube will be

$$
\begin{aligned}
\boldsymbol{F}_{\perp}= & -\left[\hat{\boldsymbol{l}} \times \nabla\left(p+B^{2} / \mu\right)\right] \times \hat{\boldsymbol{l}}+\left(\frac{(\boldsymbol{B} \cdot \nabla) \boldsymbol{B}}{\mu}\right)_{\perp} \\
& +\rho_{\mathrm{i}}(\hat{\boldsymbol{l}} \times \boldsymbol{g}) \times \hat{\boldsymbol{l}}+(\hat{\boldsymbol{l}} \times \boldsymbol{F}) \times \hat{\boldsymbol{l}} .
\end{aligned}
$$

According to Spruit (1981), the Lorentz force can be simply written as

$$
\left(\frac{(\boldsymbol{B} \cdot \nabla) \boldsymbol{B}}{\mu}\right)_{\perp}=\frac{B^{2}}{\mu} \boldsymbol{t} \text {. }
$$

We suppose that the tube is in equilibrium with its environment and the total pressure inside the tube is balanced by the external pressure, i.e.

$\nabla\left(p+B^{2} / \mu\right)=\nabla p_{\mathrm{e}}=\rho_{\mathrm{e}} \boldsymbol{g}$,

where $p_{\mathrm{e}}$ and $\rho_{\mathrm{e}}$ are the pressure and density outside the tube. With this in mind, the perpendicular component of the forces acting on the tube becomes

$$
\boldsymbol{F}_{\perp}=\frac{B^{2}}{\mu} \boldsymbol{t}+\left(\rho_{\mathrm{i}}-\rho_{\mathrm{e}}\right)(\hat{\boldsymbol{l}} \times \boldsymbol{g}) \times \hat{\boldsymbol{l}}+(\hat{\boldsymbol{l}} \times \boldsymbol{F}) \times \hat{\boldsymbol{l}} .
$$

The perpendicular component of forces acts to move the plasma mass (per unit volume) of $\left(\rho_{\mathrm{i}}+\rho_{\mathrm{e}}\right)$ in the tube and in the exterior. Therefore the equilibrium of forces in the transversal direction can be simply given as

$\left(\rho_{\mathrm{i}}+\rho_{\mathrm{e}}\right)\left(\frac{\mathrm{d} \boldsymbol{v}}{\mathrm{d} t}\right)_{\perp}=v_{\mathrm{A}}^{2} \rho_{\mathrm{i}} \boldsymbol{t}+\left(\rho_{\mathrm{i}}-\rho_{\mathrm{e}}\right)(\hat{\boldsymbol{l}} \times \boldsymbol{g}) \times \hat{\boldsymbol{l}}+(\hat{\boldsymbol{l}} \times \boldsymbol{F}) \times \hat{\boldsymbol{l}}$,

where we introduced the internal Alfvén speed $v_{\mathrm{A}}^{2}=B^{2} /\left(\mu \rho_{\mathrm{i}}\right)$. It should be mentioned that in the original derivation by Spruit (1981) the appearance of the term containing $\rho_{\mathrm{e}}$ on the left hand side of Eq. (6) was attributed to the apparent increase of tube's inertia. Combining Eqs. (1) and (6), the total equation of motion is given by

$$
\begin{array}{r}
\frac{\mathrm{d} \boldsymbol{v}}{\mathrm{d} t}=-\frac{1}{\rho_{\mathrm{i}}} \partial_{\mathrm{l}} p \hat{\boldsymbol{l}}+(\boldsymbol{g} \cdot \hat{\boldsymbol{l}}) \hat{\boldsymbol{l}}+\frac{1}{\rho_{\mathrm{i}}}(\boldsymbol{F} \cdot \hat{\boldsymbol{l}}) \hat{\boldsymbol{l}}+\frac{\rho_{\mathrm{i}}}{\rho_{\mathrm{i}}+\rho_{\mathrm{e}}} v_{\mathrm{A}}^{2} \boldsymbol{t} \\
+\frac{\rho_{\mathrm{i}}-\rho_{\mathrm{e}}}{\rho_{\mathrm{i}}+\rho_{\mathrm{e}}}(\hat{\boldsymbol{l}} \times \boldsymbol{g}) \times \hat{\boldsymbol{l}}+\frac{1}{\rho_{\mathrm{i}}+\rho_{\mathrm{e}}}(\hat{\boldsymbol{l}} \times \boldsymbol{F}) \times \hat{\boldsymbol{l}} .
\end{array}
$$

Let us write the Cartesian components of the unit vectors $\hat{\boldsymbol{l}}=$ $\left(l_{x}, l_{y}, l_{z}\right)$ and $\hat{\boldsymbol{t}}=\left(t_{x}, t_{y}, t_{z}\right)$, so the cartesian components of Eq. (7) can be written as

$$
\begin{aligned}
\left(\frac{\mathrm{d} v}{\mathrm{~d} t}\right)_{x}= & -\frac{1}{\rho_{\mathrm{i}}} \partial_{1} p l_{x}+g l_{x} l_{z}+\frac{\rho_{\mathrm{i}}}{\rho_{\mathrm{i}}+\rho_{\mathrm{e}}} v_{\mathrm{A}}^{2} t_{x}-\frac{\rho_{\mathrm{i}}-\rho_{\mathrm{e}}}{\rho_{\mathrm{i}}+\rho_{\mathrm{e}}} g l_{x} l_{z} \\
& +\frac{F}{\rho_{\mathrm{i}}+\rho_{\mathrm{e}}}+\frac{F \rho_{\mathrm{e}}}{\rho_{\mathrm{i}}\left(\rho_{\mathrm{i}}+\rho_{\mathrm{e}}\right)} l_{x}^{2}
\end{aligned}
$$




$$
\begin{aligned}
\left(\frac{\mathrm{d} v}{\mathrm{~d} t}\right)_{y}= & -\frac{1}{\rho_{\mathrm{i}}} \partial_{\mathrm{l}} p l_{y}+g l_{y} l_{z}+\frac{\rho_{\mathrm{i}}}{\rho_{\mathrm{i}}+\rho_{\mathrm{e}}} v_{\mathrm{A}}^{2} t_{y}-\frac{\rho_{\mathrm{i}}-\rho_{\mathrm{e}}}{\rho_{\mathrm{i}}+\rho_{\mathrm{e}}} g l_{y} l_{z} \\
& +\frac{F \rho_{\mathrm{e}}}{\rho_{\mathrm{i}}\left(\rho_{\mathrm{i}}+\rho_{\mathrm{e}}\right)} l_{x} l_{y}, \\
\left(\frac{\mathrm{d} \boldsymbol{v}}{\mathrm{d} t}\right)_{z}= & -\frac{1}{\rho_{\mathrm{i}}} \partial_{\mathrm{l}} p l_{z}+g l_{z}^{2}+\frac{\rho_{\mathrm{i}}}{\rho_{\mathrm{i}}+\rho_{\mathrm{e}}} v_{\mathrm{A}}^{2} t_{z}+\frac{\rho_{\mathrm{i}}-\rho_{\mathrm{e}}}{\rho_{\mathrm{i}}+\rho_{\mathrm{e}}} g\left(1-l_{z}^{2}\right) \\
& +\frac{F \rho_{\mathrm{e}}}{\rho_{\mathrm{i}}\left(\rho_{\mathrm{i}}+\rho_{\mathrm{e}}\right)} l_{x} l_{z} .
\end{aligned}
$$

These equations must be supplemented by the continuity and induction equations which can be combined into a single equation of the form

$$
\frac{\mathrm{d}}{\mathrm{d} t}\left(\frac{\rho}{B}\right)+\frac{\rho}{B}\left(\partial_{1} \boldsymbol{v}_{1}+\boldsymbol{v} \cdot \boldsymbol{t}\right)=0
$$

where $v_{1}=\boldsymbol{v} \cdot \hat{\boldsymbol{l}}$ and $\boldsymbol{t}=\partial_{1} \hat{\boldsymbol{l}}$. Now suppose that the flux tube is nearly vertical and let us denote the horizontal displacement of the tube by $\xi(z, t)$. In order to simplify the mathematics we suppose that these displacements are small. According to Spruit (1981) the components of the unit vector $\hat{\boldsymbol{l}}$ and $\hat{\boldsymbol{t}}$ can be written as

$l_{x}=\frac{\partial \xi}{\partial z}, \quad l_{z}=1+O\left(\xi^{2}\right)$,

$t_{x}=\frac{\partial^{2} \xi}{\partial z^{2}}+O\left(\xi^{2}\right), \quad t_{z}=O\left(\xi^{2}\right)$.

In addition we assume that the tube is in the $x z$-plane, so we choose $l_{y}=t_{y}=0$. Now the remaining two equations of the system (8) reduce to

$$
\begin{aligned}
\left(\frac{\mathrm{d} v}{\mathrm{~d} t}\right)_{x}= & \left(-\frac{1}{\rho_{\mathrm{i}}} \frac{\partial p}{\partial z}+g-\frac{\rho_{\mathrm{i}}-\rho_{\mathrm{e}}}{\rho_{\mathrm{i}}+\rho_{\mathrm{e}}} g\right) \frac{\partial \xi}{\partial z}+\frac{\rho_{i} v_{\mathrm{A}}^{2}}{\rho_{\mathrm{i}}+\rho_{\mathrm{e}}} \frac{\partial^{2} \xi}{\partial z^{2}} \\
& +\frac{F}{\rho_{\mathrm{i}}+\rho_{\mathrm{e}}}+\frac{F \rho_{\mathrm{e}}}{\rho_{\mathrm{i}}\left(\rho_{\mathrm{i}}+\rho_{\mathrm{e}}\right)}\left(\frac{\partial \xi}{\partial z}\right)^{2},
\end{aligned}
$$

and

$\left(\frac{\mathrm{d} v}{\mathrm{~d} t}\right)_{z}=-\frac{1}{\rho_{\mathrm{i}}} \frac{\partial p}{\partial z}+g+\frac{F \rho_{\mathrm{e}}}{\rho_{\mathrm{i}}\left(\rho_{\mathrm{i}}+\rho_{\mathrm{e}}\right)} \frac{\partial \xi}{\partial z}$.

In the above equations we restricted ourselves to linear motion only. Next we assume that the vertical displacements are small and of the same order as $\xi$. In order to obtain a closed equation, in addition, we assume that the force, $F$ acting externally on the tube is of the same order as $\xi$. Collecting terms of the same order (with respect to $\xi$ ) in the two equations we obtain that

$$
\begin{aligned}
& \left(\frac{\mathrm{d} v}{\mathrm{~d} t}\right)_{x}=\frac{\mathrm{d} v_{x}}{\mathrm{~d} t}+O\left(\xi^{2}\right)=\frac{\partial^{2} \xi}{\partial t^{2}}+O\left(\xi^{2}\right), \\
& \frac{\partial p}{\partial z}=\rho_{\mathrm{i}} g+O\left(\xi^{2}\right) .
\end{aligned}
$$

After inserting these two relations back into Eq. (11) we obtain

$\frac{\partial^{2} \xi}{\partial t^{2}}=-\frac{\rho_{\mathrm{i}}-\rho_{\mathrm{e}}}{\rho_{\mathrm{i}}+\rho_{\mathrm{e}}} g \frac{\partial \xi}{\partial z}+\frac{\rho_{\mathrm{i}} v_{\mathrm{A}}^{2}}{\rho_{\mathrm{i}}+\rho_{\mathrm{e}}} \frac{\partial^{2} \xi}{\partial z^{2}}+\frac{F}{\rho_{\mathrm{i}}+\rho_{\mathrm{e}}}$.

The above equation describes the propagation of transversal oscillations of a vertical flux tube when the oscillations are driven by an external force, $F$. Similar to Spruit (1981), the first term on the right hand side is due to stratification and is proportional to the buoyancy force, while the second term is due to the restoring force due to the magnetic tension in the tube. This equation (without the external force) has been originally derived by Lamb (1932). Equation (14) is similar to the equation derived by Spruit (his Eq. (29)) apart from the driving term on the right hand side of our equation. The propagation of kink modes described by a KG equation was studied earlier by, e.g. Musielak \& Ulmschneider (2003), Erdélyi \& Hargreaves (2008) \& Hargreaves (2008).

In what follows we are going to solve Eq. (14) for a coronal loop when the driving force is due to the incident EIT wave. Before presenting the solutions we introduce a new function, $Q$, defined as $\xi=Q \exp [\lambda z]$ and we choose the value of the parameter $\lambda$ such that all first derivatives with respect to $z$ vanish. After a straightforward calculation we obtain that when

$\lambda=g \frac{\rho_{\mathrm{i}}-\rho_{\mathrm{e}}}{2 \rho_{\mathrm{i}} v_{\mathrm{A}}^{2}}$

the governing Eq. (14) reduces to

$\frac{\partial^{2} Q}{\partial t^{2}}-\frac{\rho_{\mathrm{i}} v_{\mathrm{A}}^{2}}{\rho_{\mathrm{i}}+\rho_{\mathrm{e}}} \frac{\partial^{2} Q}{\partial z^{2}}+g^{2} \frac{\left(\rho_{\mathrm{i}}-\rho_{\mathrm{e}}\right)^{2}}{4\left(\rho_{\mathrm{i}}+\rho_{\mathrm{e}}\right) \rho_{i} v_{\mathrm{A}}^{2}} Q=\frac{F \mathrm{e}^{-\lambda z}}{\rho_{\mathrm{i}}+\rho_{\mathrm{e}}}$,

which is a nonhomogeneous Klein-Gordon (KG) equation. A nonhomogeneous $\mathrm{KG}$ equation has been also derived earlier by Rae \& Roberts (1982) and the inhomogeneous part described the effect of the external medium. In their analysis the inhomogeneous part was neglected by considering a situation where the temporal variations of the parameters outside the loop are very slow compared to changes inside the tube. For kink modes, Roberts (2004) has obtained a similar equation.

The transversal waves described by Eq. (15) will propagate with the speed given by the second term on the left hand side

$c_{\mathrm{K}}=\sqrt{\frac{\rho_{\mathrm{i}}}{\rho_{\mathrm{i}}+\rho_{\mathrm{e}}}} v_{\mathrm{A}}$

which is the kink speed. This quantity has been previously discussed within the context of wave propagation in magnetic flux tubes by, e.g. Edwin \& Roberts (1983). The coefficient of the third term has dimension of $s^{-2}$ and its square root is given by

$\omega_{\mathrm{c}}=\frac{g}{2 v_{\mathrm{A}}} \sqrt{\frac{\left(\rho_{\mathrm{i}}-\rho_{\mathrm{e}}\right)^{2}}{\left(\rho_{\mathrm{i}}+\rho_{\mathrm{e}}\right) \rho_{\mathrm{i}}}}$,

and constitutes the cut-off frequency for kink modes propagating in coronal loops. For typical coronal parameters $\left(v_{\mathrm{A}}=\right.$ $\left.900 \mathrm{~km} \mathrm{~s}^{-1}, \rho_{\mathrm{i}} / \rho_{\mathrm{e}}=10\right)$ the cut-off frequency of kink oscillations is about $0.13 \mathrm{mHz}$. With these new notations, Eq. (15) becomes

$\frac{\partial^{2} Q}{\partial t^{2}}-c_{\mathrm{K}}^{2} \frac{\partial^{2} Q}{\partial z^{2}}+\omega_{\mathrm{c}}^{2} Q=\mathcal{F}$,

where $\mathcal{F}=F \mathrm{e}^{-\lambda z} /\left(\rho_{\mathrm{i}}+\rho_{\mathrm{e}}\right)$.

Employing a normal mode analysis $\left(Q \sim \mathrm{e}^{\mathrm{i}(\omega t-k z)}\right)$ for the homogeneous part of Eq. (18), the dispersion relation of these linear waves is given as

$\omega= \pm \sqrt{k^{2} c_{\mathrm{K}}^{2}+\omega_{\mathrm{c}}^{2}}$

Due to the particular $k$-dependence of the dispersion relation waves are dispersive, i.e. waves with larger wavelength 
(shorter $k$ ) propagating faster. The group speed of these waves is given as

$\partial \omega / \partial k= \pm \frac{k c_{\mathrm{K}}^{2}}{\sqrt{k^{2} c_{\mathrm{K}}^{2}+\omega_{\mathrm{c}}^{2}}}$,

so, waves with smaller wave number will have smaller group speed, the maximum of the group speed (at $k \rightarrow \infty$ ) being $c_{\mathrm{K}}$.

Equation (18) has been studied in the context of pulse propagation in the solar photosphere and chromosphere (see, e.g. Roberts \& Webb 1978; Rae \& Roberts 1982; Kalkofen et al. 1994; Sutmann et al. 1998; Hassan \& Kalkofen 1999; Musielak \& Ulmschneider 2001, 2003; Hindman \& Jain 2008; Erdélyi \& Hargreaves 2008). The solution of the KG equation represents the propagation of a wave with the speed $c_{\mathrm{K}}$ followed by a wake oscillating with the frequency $\omega_{\mathrm{c}}$.

An extension of the $\mathrm{KG}$ equation has been discussed by Ballai et al. (2006) when the dissipation (kinematic viscosity in their analysis) modified the $\mathrm{KG}$ equation into a Klein-Gordon-Burgers equation where the dissipative term was given as a mixed (space and time) derivative.

In what follows we present an analytical solution to Eq. (18) in the most general form and particular solutions will be deducted. Let us suppose that the boundary and initial conditions used for solving Eq. (18) are given by

$Q(0, t)=Q(L, t)=0$,

$Q(z, 0)=u_{1}(z), \quad \frac{\partial Q}{\partial t}(z, 0)=u_{2}(z)$.

First we apply the Laplace transform to Eq. (18) and we obtain

$c_{\mathrm{K}}^{2} \frac{\partial^{2} \Psi}{\partial z^{2}}-\left(s^{2}+\omega_{\mathrm{c}}^{2}\right) \Psi=-\Phi-s u_{1}(z)-u_{2}(z)$,

where $\Psi(z, s)$ and $\Phi(z, s)$ are the Laplace transforms of the functions $Q(z, t)$ and $\mathcal{F}(z, t)$ defined as

$\Psi(z, s)=\int_{0}^{\infty} Q(z, t) \mathrm{e}^{-s t} \mathrm{~d} t, \quad \Phi(z, s)=\int_{0}^{\infty} \mathcal{F}(z, t) \mathrm{e}^{-s t} \mathrm{~d} t$.

Given the nature of the boundary conditions we further apply a finite Fourier sine transform defined as

$F_{s}[f(x)]=\frac{1}{L} \int_{0}^{L} f(x) \sin \alpha_{n} x \mathrm{~d} x$,

where we introduced

$\alpha_{n}=\frac{n \pi}{L}$.

After applying the Fourier sine transform we obtain

$\left(s^{2}+\omega_{\mathrm{c}}^{2}+c_{\mathrm{K}}^{2} \alpha_{n}^{2}\right) \bar{\Psi}(n, s)=\bar{\Phi}(n, s)+s \bar{u}_{1}(n)+\bar{u}_{2}(n)$,

where the functions with an overline represent the Fourier transformed functions. From Eq. (22) we obtain that

$\bar{\Psi}(n, s)=\frac{\bar{\Phi}(n, s)+s \bar{u}_{1}(n)+\bar{u}_{2}(n)}{s^{2}+\omega_{\mathrm{c}}^{2}+c_{\mathrm{K}}^{2} \alpha_{n}^{2}}$.

Now we apply an inverse Fourier transform which results in

$$
\begin{aligned}
\Psi(z, s)= & \frac{2}{L} \sum_{n=1}^{\infty} \frac{\sin \alpha_{n} z}{s^{2}+\omega_{\mathrm{c}}^{2}+c_{\mathrm{K}}^{2} \alpha_{n}^{2}}\left[\int_{0}^{L} \bar{\Phi}(\zeta, s) \sin \alpha_{n} \zeta \mathrm{d} \zeta\right. \\
& \left.+s \int_{0}^{L} \bar{u}_{1}(\zeta) \sin \alpha_{n} \zeta \mathrm{d} \zeta+\int_{0}^{L} \bar{u}_{2}(\zeta) \sin \alpha_{n} \zeta \mathrm{d} \zeta\right]
\end{aligned}
$$

In order to obtain $Q(z, t)$, we need to apply an inverse Laplace transform to the function $\Psi(z, s)$. When calculating this transform we will take into account the results of the convolution theorem, i.e.

$\mathcal{L}^{-1}\{\bar{f}(s) \bar{g}(s)\}=\bar{f} * \bar{g}=\int_{0}^{t} f(\tau) g(t-\tau) \mathrm{d} \tau$,

as well as the inverse Laplace transforms of the quantities

$\mathcal{L}^{-1}\left(\frac{1}{s^{2}+\omega_{\mathrm{c}}^{2}+c_{\mathrm{K}}^{2} \alpha_{n}^{2}}\right)=\frac{\sin \sqrt{\omega_{\mathrm{c}}^{2}+c_{\mathrm{K}}^{2} \alpha_{n}^{2}} t}{\sqrt{\omega_{\mathrm{c}}^{2}+c_{\mathrm{K}}^{2} \alpha_{n}^{2}}}=\frac{1}{\omega_{n}} \sin \omega_{n} t$,

and

$\mathcal{L}^{-1}\left(\frac{s}{s^{2}+\omega_{\mathrm{c}}^{2}+c_{\mathrm{K}}^{2} \alpha_{n}^{2}}\right)=\cos \sqrt{\omega_{\mathrm{c}}^{2}+c_{\mathrm{K}}^{2} \alpha_{n}^{2}} t=\cos \omega_{n} t$,

with $\omega_{n}=\sqrt{\omega_{\mathrm{c}}^{2}+c_{\mathrm{K}}^{2} \alpha_{n}^{2}}$ being the natural frequency of the loop and the mode corresponding to $n=0$ being the cut-off frequency. It is interesting to note that the ratio of periods corresponding to the fundamental mode $(n=1)$ and the first harmonic $(n=2)$ is given by

$$
\frac{T_{1}}{2 T_{2}}=\frac{1}{2} \sqrt{\frac{\omega_{\mathrm{c}}^{2}+4 c_{\mathrm{K}}^{2} \pi^{2} / L^{2}}{\omega_{\mathrm{c}}^{2}+c_{\mathrm{K}}^{2} \pi^{2} / L^{2}}} .
$$

Due to the presence of the cut-off frequency this period ratio is not 1 but is slightly smaller, however it can approach the observed period ratio (Verwichte et al. 2004; McEwan et al. 2006) if the cut-off is made unrealistically high. For typical coronal and loop conditions, the natural periods of a loop of $L=200 \mathrm{Mm}$ and $c_{\mathrm{K}}=1000 \mathrm{~km} \mathrm{~s}^{-1}$ are $400 \mathrm{~s}, 200 \mathrm{~s}$, and $133 \mathrm{~s}$, respectively while for a $300 \mathrm{Mm}$ loop these periods will be in a ratio $600 / 300 / 200$. If the length is fixed at $200 \mathrm{Mm}$ and let the kink speed to be $1100 \mathrm{~km} \mathrm{~s}^{-1}$ the periods of the first three modes will be 364/182/121. It should be pointed out that Eq. (25) is similar to the findings in McEwan et al. (2006) though their equation was written for slow standing modes (see their Eq. (24)). However, observers have not reported harmonics for slow waves whereas reports on higher harmonics for kink modes are in abundance.

In the light of these relations, the inverse Laplace transform of, e.g. the first term in Eq. (24) will be of the form

$\mathcal{L}^{-1}\left(\frac{\bar{\Phi}(\zeta, s)}{s^{2}+\omega_{n}^{2}}\right)=\frac{1}{\omega_{n}} \int_{0}^{t} \Phi(\zeta, \tau) \sin \left(\omega_{n}(t-\tau)\right) \mathrm{d} \tau$.

Applying a term-by-term inversion to the function $\Psi(z, s)$ given by Eq. (24) we obtain

$$
\begin{aligned}
& Q(z, t)=\frac{2}{L}\left\{\sum_{n=1}^{\infty} \frac{\sin \alpha_{n} z}{\omega_{n}} \int_{0}^{L} \sin \alpha_{n} \zeta \mathrm{d} \zeta \int_{0}^{t} \Phi(\zeta, \tau)\right. \\
& \times \sin \left(\omega_{n}(t-\tau)\right) \mathrm{d} \tau+\sum_{n=1}^{\infty} \sin \alpha_{n} z\left[\cos \omega_{n} t \int_{0}^{L} u_{1}(\zeta) \sin \alpha_{n} \zeta \mathrm{d} \zeta\right. \\
& \left.\left.\quad+\frac{\sin \omega_{n} t}{\omega_{n}} \int_{0}^{L} u_{2}(\zeta) \sin \alpha_{n} \zeta \mathrm{d} \zeta\right]\right\}
\end{aligned}
$$

The solution of the nonhomogeneous Klein-Gordon equation given by Eq. (27) can be simplified once the forms of the functions $u_{1}$ and $u_{2}$ are known. In what follows we will discuss a few 
particular cases and will investigate the possibility of generating oscillations in coronal loops triggered by an incident wave modelling the coronal global EIT wave.

The simplest particular case is when we have zero initial conditions, i.e. $u_{1}(z)=u_{2}(z)=0$, and

$\mathcal{F}(z, t)=f(t) \delta\left(z-z_{0}\right)$,

where $\delta(z)$ is the Dirac-delta function. In this case the solution of the inhomogeneous $\mathrm{KG}$ equation is given by

$$
\begin{aligned}
Q(z, t)= & \frac{2}{L^{2}} \sum_{n=1}^{\infty} \frac{\sin \alpha_{n} z \sin \alpha_{n} z_{0}}{\omega_{n}} \\
& \times \int_{0}^{t} f(\tau) \sin \left(\omega_{n}(t-\tau)\right) \mathrm{d} \tau,
\end{aligned}
$$

where we used the property that

$\delta\left(z-z_{0}\right) \sin \alpha_{n} z= \begin{cases}\sin \alpha_{n} z_{0}, & \text { if } 0 \leq z_{0} \leq L \\ 0, & \text { otherwise. }\end{cases}$

When deriving Eq. (29) we took into account that the $\delta$-function has a dimension of $L^{-1}$ and a variable change of the form $\tilde{z}=$ $z / L$ and $\tilde{z}_{0}=z_{0} / L$ is needed. The extra $L$ in the denominator of Eq. (29) arises after we apply the property that $\delta\left[L\left(\tilde{z}-\tilde{z}_{0}\right)\right]=$ $L^{-1} \delta\left(\tilde{z}-\tilde{z}_{0}\right)$. If we further assume that $f(\tau)=\delta(\tau)$ (i.e. the source consists of an impulse acting at $z=z_{0}$ ) the solutions describing the oscillations in a fixed-ends loop is given by

$$
\begin{aligned}
Q(z, t)= & 2 c_{\mathrm{K}} L \\
& \times \sum_{n=1}^{\infty} \frac{\sin \alpha_{n} z \sin \alpha_{n} z_{0}}{\omega_{n}} \sin \omega_{n} t=G\left(z, t / z_{0}\right),
\end{aligned}
$$

which constitute the Green function for the coronal loop modelled as a straight structure fixed at $z=0, L$. Once the Green function is known, the solution of the inhomogeneous KG equation for an arbitrary external action $\mathcal{F}(z, t)$ can be written as

$Q(z, t)=\int_{0}^{L} \mathrm{~d} z^{\prime} \int_{0}^{t} G\left(z, t-\tau / z^{\prime}\right) \mathcal{F}\left(z^{\prime}, \tau\right) \mathrm{d} \tau$.

The present analysis does not include any information about the radius of the tube geometrical or the internal structure of the tube and external magnetic field, factors which could be important. However, it is easy to estimate the magnitude of the external force required to induce oscillations in the tube. The magnetic tension force in the tube with constant circular cross-section is $\left(B^{2} / \mu\right) \pi R^{2}$ where $R$ is the constant radius of the tube. The external force must be at least as large as the tension of the tube. Writing a simple force equilibrium equation in transversal direction to the axis fo the tube we obtain that the external force acting on the tube in a point $z_{0}$ along the tube has to be larger than

$\frac{B^{2}}{\mu} \pi R^{2}\left[\frac{1}{\left(1+\lambda_{\mathrm{e}}^{2} z_{0}^{2}\right)^{1 / 2}}+\frac{1}{\left(1+\lambda_{\mathrm{e}}^{2}\left(L-z_{0}\right)^{2}\right)^{1 / 2}}\right]$,

where $1 / \lambda_{\mathrm{e}}$ is the maximum displacement of the tube and is given by (see, e.g. Edwin \& Roberts 1983)

$\lambda_{\mathrm{e}}=k \sqrt{\frac{c_{\mathrm{K}}^{2}-c_{\mathrm{Se}}^{2}}{c_{\mathrm{Se}}^{2}}}$,

with $c_{\text {Se }}$ being the sound speed in the magnetic free region outside the coronal loop and $k=\pi n / L$ is the longitudinal wavenumber. For a loop length of $200 \mathrm{Mm}$ and $c_{\mathrm{K}}=1000 \mathrm{~km} \mathrm{~s}^{-1}$,
$c_{\mathrm{Se}}=200 \mathrm{~km} \mathrm{~s}^{-1}$ we obtain a maximum displacement of the fundamental mode of $12.9 \mathrm{Mm}$.

In the following section we are going to discuss a few particular cases referring to the nature of the driver and find the equation giving the transversal displacement of the loop as given by Eq. (27).

\section{Drivers of particular form}

The discussion of these separate particular cases is needed as the true nature of EIT waves is not known. As specified before, the force on the right hand side of Eq. (14) is the force which acts on the coronal loop and represents the effect of the incident EIT wave on the coronal loop. Obviously it is difficult to estimate the value (or the direction) of this force, however, some estimations can be made (see also the end of the previous section). If we suppose that the entire energy of the EIT wave $\left(E_{\mathrm{EIT}}\right)$ is converted into inducing oscillations of the loop, the energy of the EIT wave will work toward displacing the loop. Therefore we can write that

$E_{\mathrm{EIT}}=\frac{F}{\lambda_{\mathrm{e}}}$.

Obviously the energy of EIT waves is quantity which cannot be directly measured however, previous indirect estimations (Ballai 2007) show that these energies are in the range of $10^{16}-10^{19} \mathrm{~J}$.

\subsection{Harmonic drivers}

Let us suppose that the EIT wave is a wave and its action of the coronal loop is described by a force of the form

$\mathcal{F}=E_{\mathrm{EIT}} \lambda_{\mathrm{e}} \frac{\delta\left(z-z_{0}\right) \mathrm{e}^{-\lambda z} \mathrm{e}^{\mathrm{i} \omega_{\mathrm{EIT}} t}}{\rho_{\mathrm{i}}+\rho_{\mathrm{e}}}$

where $\omega_{\text {EIT }}$ is the frequency of EIT waves. This form of the externally acting force is inserted back into Eq. (27), yielding

$$
\begin{aligned}
Q(z, t)= & -\frac{4 E_{\mathrm{EIT}} \lambda_{\mathrm{e}}}{L^{3}\left(\rho_{\mathrm{i}}+\rho_{\mathrm{e}}\right)} \sum_{n=1}^{\infty} \frac{\mathrm{e}^{-\lambda z_{0}} \sin \alpha_{n} z \sin \alpha_{n} z_{0}}{\left(\omega_{\mathrm{EIT}}^{2}-\omega_{n}^{2}\right)} \\
& \times\left\{\left[\sin \left(\frac{\omega_{n}+\omega_{\mathrm{EIT}}}{2} t\right) \sin \left(\frac{\omega_{n}-\omega_{\mathrm{EIT}}}{2} t\right)\right]\right. \\
& \left.+\mathrm{i}\left(\omega_{\mathrm{EIT}} \sin \omega_{n} t-\omega_{n} \sin \omega_{\mathrm{EIT}} t\right)\right\} .
\end{aligned}
$$

The presence of the $\sin \alpha_{n} z_{0}$ in the expression $Q(z, t)$ simply means that the amplitude of generated oscillations will depend on the height (along the loop) where the EIT wave interacts with the loop. The maximum amplitude of oscillations (in the case of modes with odd $n$ ) will be reached when the EIT wave hits the top of the loop, i.e. the interaction occurs at $z_{0}=L / 2$. In this case there will be no modes generated corresponding to an even $n$ (for instance for an interaction of this type we will not have first harmonics present in the loop). This statement supports the conclusions drawn by Ballai (2007) where a list with possible factors which can influence the characteristics of loop oscillations was given. The singularity in the denominator of Eq. (33) is just apparent, its effect is balanced by the numerator. If we concentrate only on the real part of Eq. (33), it is also obvious that the resulting signal will not have a well defined standing mode pattern, instead the oscillations will be a superposition of different oscillations. The real part of Eq. (33) is numerically represented in Fig. 2 (in all subsequent figures we will always use the real part). The period of the driver EIT wave is left to vary between 50 and $800 \mathrm{~s}$. 


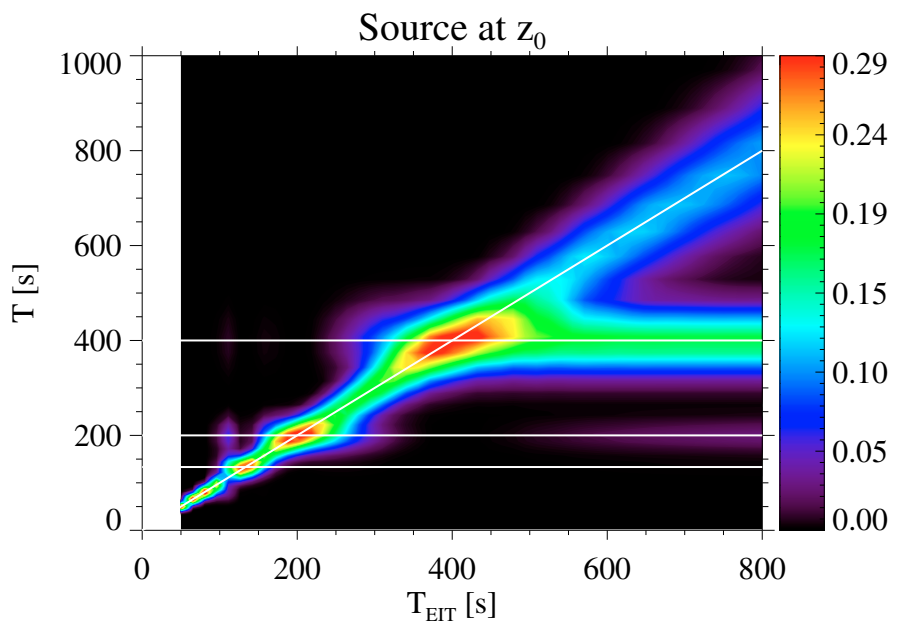

Fig. 2. The periods of oscillations generated by an EIT wave acting at $z=z_{0}$. The period of the driver is changed in the interval 50 to 800 s. The horizontal lines represent the natural periods of the loop, i.e. $T_{n}=2 \pi / \omega_{n}$. The inclined line corresponds to the periods of the EIT wave, the driver of the oscillations in the coronal loop.

The pattern of oscillations which can be generated in the coronal loop depends on the characteristics of the driver. For each period of the driver (in between 50 and $800 \mathrm{~s}$ ) a wavelet analysis has been carried out for the temporal part of Eq. (33). The power of the signal has been summed up inside the cone of influence set at a confidence level of $95 \%$ and the results are shown in Fig. 2 which is color-coded, the red color corresponds to the highest power, while the black color represents the lowest power. Depending on the period of the EIT wave, various oscillation modes can be excited. For the example shown in Fig. 2, the loop has a length of $200 \mathrm{Mm}$ and a kink speed of $1000 \mathrm{~km} \mathrm{~s}^{-1}$. The natural periods corresponding to these values are in a ratio of $400 / 200 / 133$, values represented by the horizontal lines.

Let us consider a driver which has a period larger than $600 \mathrm{~s}$. In this case, the modes which can be excited will be the fundamental mode (corresponding to $400 \mathrm{~s}$ ) and the first harmonic but with a very low power. The oscillation pattern of the driver is still present but much weaker than the oscillation of the fundamental mode. As the period of the driver becomes smaller, other harmonics can be excited. For a period between 200 and $400 \mathrm{~s}$ the pattern of the driver is preserved (see the inclined bright direction) but a considerable amount of the fundamental mode and first harmonic can be generated. Higher harmonics are also present but their power is very small. For a period of less than $200 \mathrm{~s}$ the dominant oscillations will be the first and second harmonics, while the fundamental mode is extremely weak. The red regions correspond to the cases when the period of the driver matches (or is very close) to one of the natural periods of the loop. In that case there is a resonance between the driver and the coronal loop.

In reality, however, if the EIT wave is an oscillating front colliding with the coronal loop, then the interaction occurs not only in one point, but in two, symmetrically situated from the ends of the loop. Let us suppose now that the driver is a front which interacts with the loop at the same time in two points, at $z_{0}$ and at $L-z_{0}$. In this case, the driver will have the form

$\mathcal{F}=E_{\text {EIT }} \lambda_{\mathrm{e}} \frac{\left[\delta\left(z-z_{0}\right)+\delta\left(z-L+z_{0}\right)\right] \mathrm{e}^{-\lambda z}}{\rho_{\mathrm{i}}+\rho_{\mathrm{e}}} \mathrm{e}^{\mathrm{i} \omega_{\mathrm{EIT}} t}$.

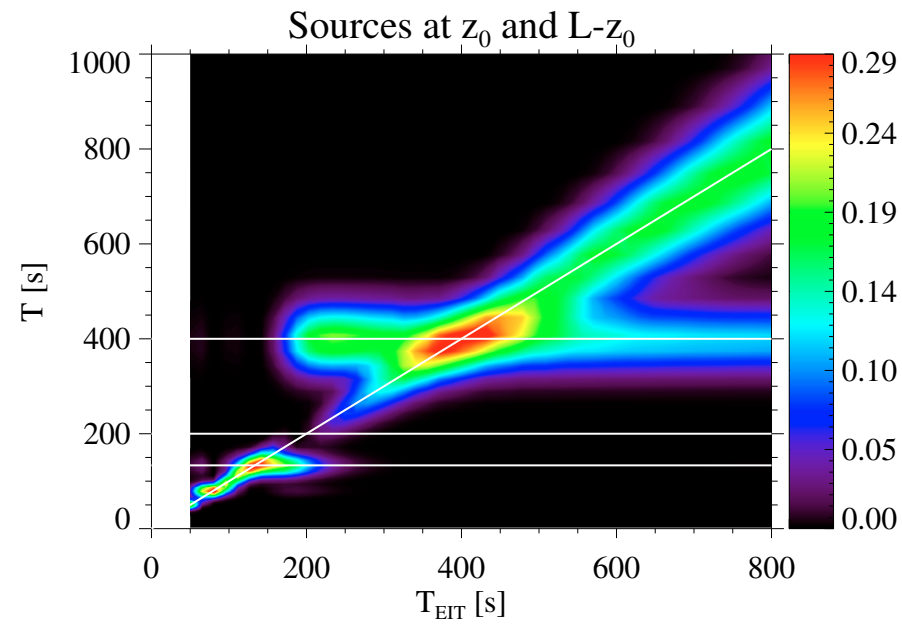

Fig. 3. The same as in Fig. 2 but now the oscillations are generated by an EIT wave interacting with the coronal loop at $z=z_{0}$ and $z=L-z_{0}$.

For this expression the resulting oscillations will be described by a similar function as given by Eq. (33) but now the spatial dependence will contain in the numerator the expression

$\mathrm{e}^{\lambda z_{0}} \sin \alpha_{n} z_{0}+\mathrm{e}^{-\lambda\left(L-z_{0}\right)} \sin \alpha_{n}\left(L-z_{0}\right)$.

This situation can be achieved if the front of the EIT wave is perfectly perpendicular to the coronal loop. For this particular driver the corresponding period-diagram is shown in Fig. 3. According to expectations, in this case no modes corresponding to an even $n$ will be excited. In contrast to the first case, for driver's period larger than the period corresponding to the first natural period (400 s) the EIT wave will excite modes which will carry predominantly the characteristic of the driver and in a smaller quantity the properties of the fundamental mode. No first harmonic can be generated, instead for a narrow range of the driver's period, (a small interval around $200 \mathrm{~s}$ ) the oscillations will comprise addition from the fundamental mode and the second harmonic.

In reality it is more likely that the front of the incident EIT wave is not completely perpendicular to the axis of the loop, now the two interaction points between the loop and EIT wave will separated by a delay time, i.e. the time required for the front to reach the other half of the loop. The delay time can be easily calculated (see for details Ballai 2007) and depends on the length of the loop, the speed of propagation of the EIT wave and the attack angle, i.e. the angle the front of the EIT wave makes with the vertical plane of the coronal loop. In this case, the acting force will have a spatial and temporal dependence of the form

$\delta\left(z-z_{0}\right) \mathrm{e}^{\mathrm{i} \omega_{\text {EIT }} t}+\delta\left(z-L+z_{0}\right) \mathrm{e}^{\mathrm{i} \omega_{\text {EIT }}\left(t-T_{\mathrm{d}}\right)} H\left(t-T_{\mathrm{d}}\right)$,

where $T_{\mathrm{d}}$ is the delay time and $H(t)$ is the Heaviside step function. After inserting this form back into Eq. (27) we obtain that the modified transversal displacement of the coronal loop is of the form

$$
\begin{aligned}
Q(z, t)= & -\frac{4 E_{\mathrm{EIT}} \lambda_{\mathrm{e}}}{L^{3}\left(\rho_{\mathrm{i}}+\rho_{\mathrm{e}}\right)} \sum_{n=1}^{\infty} \frac{\sin \alpha_{n} z}{\left(\omega_{\mathrm{EIT}}^{2}-\omega_{n}^{2}\right)} \\
& \times\left\{\mathrm{e}^{-\lambda z_{0}} \sin \alpha_{n} z_{0}\left[\cos \omega_{n} t-\cos \omega_{\mathrm{EIT}} t\right]-\mathrm{e}^{-\lambda\left(L-z_{0}\right)}\right. \\
& \left.\times \sin \alpha_{n}\left(L-z_{0}\right)\left[\cos \omega_{n}\left(t-T_{\mathrm{d}}\right)-\cos \omega_{\mathrm{EIT}}\left(t-T_{\mathrm{d}}\right)\right]\right\} .
\end{aligned}
$$

As a particular case we have chosen the situation when the delay time corresponds to an integer number of EIT wave's period. In the case of two external forces acting in phase upon the coronal loop the possible modes appearing in the coronal loop are 


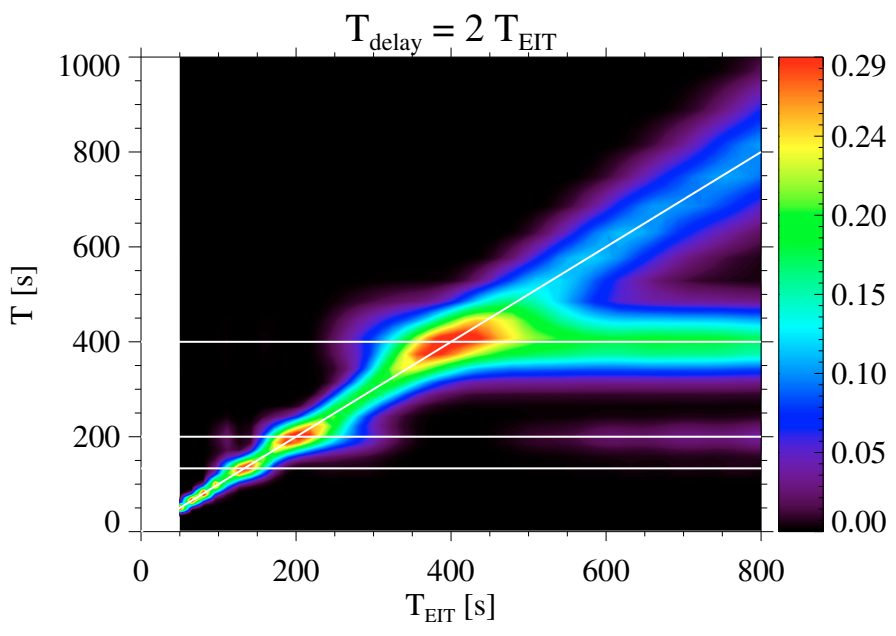

Fig. 4. The same as in Fig. 3 but now the oscillations are generated by an EIT wave acting at $z=z_{0}$ and $z=L-z_{0}$ and the second interaction is delayed by a time corresponding to the double of EIT waves' period.

shown in Fig. 4. It is obvious that the period of generated oscillations will contain the period of the EIT wave as the strongest component. For periods larger than the natural period of the fundamental mode the generated oscillation will be dominated by the period of the fundamental mode with a weaker signal resembling the characteristics of the driver and a very weak period corresponding to the first harmonic. For periods of the driver situated between the natural periods of the loop, the possibility of mode generation goes parallel with the case explained in the case of a single driver. It can be easily shown that the distribution of possible periods in the coronal loop is similar even when the delay time is not an integer number of EIT waves' period.

\subsection{Non-harmonic driver}

EIT waves have also been explained in terms of a non-wave feature (i.e. not having a harmonic behaviour). In this context we could list the models proposed by, e.g. Delanée (2000) and Attrill et al. (2007) where EIT waves were associated with deformations and evolutions of magnetic fields resulted after the release of the CME. In order to include this possible explanation of EIT waves, let us suppose an external force acting on the coronal loop of the form

$\mathcal{F}=E_{\text {EIT }} \lambda_{\mathrm{e}} \frac{\mathrm{e}^{-\lambda_{z}}\left[H\left(z-z_{0}\right)-H\left(z-z_{0}^{\prime}\right)\right]}{\rho_{\mathrm{i}}+\rho_{\mathrm{e}}} \delta(t)$,

which means that the external driving force is represented by a finite width $\left(\left|z_{0}-z_{0}^{\prime}\right|\right)$ front which has no temporal component other than a Dirac-delta function. If the form of external force given by Eq. (35) is inserted back into Eq. (27), we obtain that the temporal part of the transversal displacement of the magnetic tube modelling a coronal loop is given by

$$
\int_{0}^{t} \delta\left(t^{\prime}\right) \sin \omega_{n}\left(t-t^{\prime}\right) \mathrm{d} t^{\prime} \sim \sin \omega_{n} t
$$

which means that this form of the EIT wave (a single finite width front) will produce oscillations in the coronal loop at the natural frequency of the loop only.

It is possible that the two forms of the external driver (the harmonic and non-harmonic) treated here coexist in the sense that they are the manifestation of the same phenomenon but at different distance from the source, therefore a more careful analysis will be needed in the future.

\section{Conclusions}

The generation and propagation of oscillations in coronal loops modelled by a straight magnetic cylinder with fixed ends is studied when the coronal loop is under the effect of an EIT wave, as a driver. We found that if all forces acting on the flux tube are taken into account, the governing equation describing the propagation of standing transversal (kink) waves is described by an inhomogeneous Klein-Gordon-type equation and the inhomogeneous part of the equation is represented by the force (over a unit volume) by EIT waves. The evolutionary equation contains information about the propagation speed of waves (here the kink speed) and the cut-off frequency of kink modes. The cut-off value is determined by the densities inside/outside the loop and the Alfvén speed (i.e. magnetic field).

Using the combined Laplace and Fourier sine transform techniques, the governing equation is solved, such that the solution takes into account general initial and boundary conditions.

Particular solutions have been found in the case of an EIT wave considered first as a wave with a frequency $\omega_{\mathrm{EIT}}$, and later as a shock wave with finite front thickness (a non-harmonic driver). The results show that in case of a non-harmonic driver the periods of generated modes always belong to the natural periods of the loop. On the other hand, in the case of a periodic driver - for an arbitrary period of the driver - there will be a mixture of standing modes which could explain on the observed period ratio. The analysis carried out here for different type of drivers show that the generated oscillations will carry predominantly information about the driver rather than the loop itself.

The oscillations described in this paper were all modelled in the framework of ideal MHD. In reality coronal loop oscillations are observed to decay relatively rapidly and several mechanisms have been proposed to explain this damping (Ruderman \& Roberts 2005; Terradas et al. 2005, 2007; Selwa et al. 2007). The inclusion of a dissipative (or energy lost) mechanism in the present model will be addressed in a future study. It could be possible that in the case of a loop oscillating as a whole in the kink mode, the friction with the environment could be also an important factor whose inclusion in the model could result in a possible explanation of the damping of these oscillations.

The present study can be further extended to the case when the external EIT wave acts not only on a single magnetic loop but on a system of adjacent loops. In this case the primary displacement of the first coronal loop (generated by the incoming EIT wave) will be the driver for the oscillations in the second loop (and so on), leading to coupled loop oscillations. In order to describe a realistic loop, the present model is going to be expanded to consider the effect of an external magnetic field. It is expected that the presence of this field will generate an additional force which will tend to suppress the oscillation of the tube.

Acknowledgements. I.B. acknowledges the financial support by NFS Hungary (OTKA, K67746). I.B. and A.M. were supported by The National University Research Council Romania (CNCSIS-PN-II/531/2007). M.D. acknowledges the support from STFC.

\section{References}

Arregui, I., Terradas, J., Oliver, R., \& Ballester, J. L. 2008, ApJ, 674, 1179 Aschwanden, M. J., Fletcher, L., Schrijver, C. J., \& Alexander, D. 1999, ApJ, 520,880 
Attrill, G. D. R., Harra, L. K., van Driel-Gesztelyi, L., Démoulin, P., \& Wüsler, J.-P. 2007a, Astron. Nachr., 328, 760

Attrill, G. D. R., Harra, L. K., van Driel-Gesztelyi, L., \& Démoulin, P. 2007b, ApJ, 656, L101

Ballai, I. 2007, Sol. Phys., 247, 177

Ballai, I., Erdélyi, R., \& Pintér, B. 2005, ApJ, 633, L145

Ballai, I., Erdélyi, R., \& Hargreaves, J. 2006, Phys. Plasmas, 13, 042108

Banerjee, D., Erdélyi, R., Ramon, O., \& O’Shea, E. 2007, Sol. Phys., 246, 3

Delanée, C. 2000, ApJ, 545, 512

Edwin, P., \& Roberts, B. 1983, Sol. Phys., 88, 179

Erdélyi, R., \& Hargreaves, J. 2008, A\&A (in press)

Hargreaves, J. 2008, Ph. D. Thesis, University of Sheffield, 84

Hasan, S. S., \& Kalkofen, W. 1999, ApJ, 519, 899

Hindman, B. W., \& Jain, R. 2008, ApJ, 677, 899

Kalkofen, W., Rossi, P., Bodo, G., \& Massaglia, S. 1994, A\&A, 284, 976

Lamb,H. 1932, Hydrodynamics (Cambridge University Press), 138

Long, D. M., Gallagher, P. T., McAteer, R. T. J., \& Bloomfield, D. S. 2008, ApJ, 680,81

McEwan, M. P., Donnelly, G. R., Diaz, A. J., \& Roberts, B. 2006, A\&A, 460, 893

Musielak, Z. E., \& Ulmschneider, P. 2001, A\&A, 370, 541
Musielak, Z. E., \& Ulmschneider, P. 2003, A\&A, 400, 1057

Nakariakov, V. M., Ofman, L., DeLuca, E. E., Roberts, B., \& Davila, J. M. 1999 , Science, 285,862

Rae, I. C., \& Roberts, B. 1982, ApJ, 256, 761

Roberts, B. 2004, in Waves, oscillations and small-scale transient events in the solar atmosphere: a joint view from SOHO and TRACE, ESA SP-547, 1

Roberts, B., \& Webb, A. R. 1978, Sol. Phys., 56, 5

Roberts, B., Edwin, P. M., \& Benz, A. O. 1984, ApJ, 279, 857

Ruderman, M. S., \& Roberts, B. 2002, ApJ, 577, 475

Selwa, M., Murawski, K., Solanki, S. K., \& Wang, T. J. 2007, A\&A, 462, 1127 Spruit, H.C 1981, A\&A, 98, 155

Sutmann, G., Musielak, Z. E., \& Ulmschneider, P. 1998, A\&A, 340, 556

Terradas, J., Oliver, R., \& Ballester, J. L. 2005, A\&A, 441, 371

Terradas, J., Andries, J., \& Goossens, M. 2007, Sol. Phys., 246, 231

Thompson, B. J., Gurman, J. B., Neupert, W. M., et al. 1999, ApJ, 517, 151

Verth, G., \& Erdélyi, R. 2008, A\&A, 486, 1015

Verth, G., van Doorselaere, T., Erdélyi, R., \& Goossens, M. 2007, A\&A, 457, 341

Verwichte, E., Nakariakov, V. M., Ofman, L., \& Deluca, E. E. 2004, Sol. Phys., 233,77

Wills-Davey, M. J., \& Thompson, B. J. 1999, Sol. Phys., 190, 467 